\title{
A importância da espiritualidade como base para os cuidados paliativos a pacientes ontológicos terminais
}

The importance of spirituality as a basis for palliative care for terminal oncological patients

La importancia de la espiritualidad como base de los cuidados paliativos para pacientes ontológicos terminales

Jorge Davi de Sousa e Silva ORCID: https://orcid.org/0000-0003-3157-1108 Centro Universitário UniFacid/Wyden, Brasil

E-mail: jddavisilva123@gmail.com

Nagila Iane Pacheco

ORCID: https://orcid.org/0000-0002-2836-1639 Centro Universitário UniFacid/Wyden, Brasil E-mail: nagilaiane@hotmail.com

Gabriel de Sousa Carneiro

ORCID: https://orcid.org/0000-0002-0881-7870 Faculdade Mauricio de Nassau, Brasil

E-mail: gabrieldesousa2735@gmail.com

Danielle Costa Lopes

ORCID: https://orcid.org/0000-0002-7382-1323 Centro Universitário UniFacid/Wyden, Brasi E-mail: dany197_@hotmail.com

Diego Rodrigues Pessoa

ORCID: https://orcid.org/0000-0002-8981-673X Universidade Federal do Piauí, Brasil

E-mail: fisio.diegorodrigues@gmail.com

Ivanira Vieira Loiola Coutinho ORCID: https://orcid.org/0000-0003-1877-1885 Centro Universitário UniFacid/Wyden, Brasil

E-mail: Ivanirafarma@gmail.com

Yago Thierry Rodrigues Cruz

ORCID: https://orcid.org/0000-0003-4131-6688 Centro Universitário UniFacid/Wyden, Brasil

E-mail: Yagorodrigues1414@gmail.com

Adryana Ryta Ribeiro Sousa Lira ORCID: https://orcid.org/0000-0002-1639-3369 Centro Universitário UniFacid/Wyden, Brasil E-mail: dryribeiro22@gmail.com

Alciene Pacheco da Silva

ORCID: https://orcid.org/0000-0003-4117-2792

Universidade Federal do Piauí, Brasil

E-mail: alcienepacheco@ufpi.edu.br

Guilherme Reis Nunes Camarço

ORCID: https://orcid.org/0000-0002-9450-6314

Centro Universitário UniFacid/Wyden, Brasil

E-mail: guilherme_reis76@hotmail.com

Leandro Ferreira Frade Soares

ORCID: https://orcid.org/0000-0003-1499-431X

Universidade Federal de Pernambuco, Brasil

E-mail: leandro_frade30@hotmail.com

Natalia de Assis Rocha

ORCID: https://orcid.org/0000-0002-4761-649X

Centro Universitário UniFacid/Wyden, Brasil

E-mail: Nataaliaassisrochaa@hotmail.com

Lyslly Rhanny Soares De Deus

ORCID: https://orcid.org/0000-0003-4367-5609

Centro Universitário UniFacid/Wyden, Brasil E-mail: lysllyrhanny@ hotmail.com

Luiza Aragão Paiva Pires Ferreira Mendes ORCID: https://orcid.org/0000-0002-4017-6102

Centro Universitário UniFacid/Wyden, Brasil E-mail: luizamendes0412@gmail.com 


\begin{abstract}
Resumo
Introdução: O CA é um problema de saúde pública mundial, que afeta indiscriminadamente os cidadãos sem restrição de idade, sexo e raça. O número de eivados e óbitos por essa doença multiplicou no decorrer dos anos, esse fato acarretou-se por múltiplas causas. Quando os processos terapêuticos não apresentam devidos resultados diante da evolução da doença, assim o quadro torna-se irreversível, nesse contexto, a equipe multidisciplinar de saúde deverá provir CP. Objetivo: Essa pesquisa tratou-se de uma revisão literária que expôs os cuidados paliativos competentes a Terapia Ocupacional na abordagem de pessoas em estado ontológico terminal e sua aplicação em consonância com a espiritualidade do indivíduo. Metodologia: Trata-se de uma revisão bibliográfica construída a partir de artigos coletados de bancos de dados online, especificamente o INCA, SCIELO, PUBMED e Google School. Utilizaram-se artigos dos anos de 2016 a 2022, utilizou no total 24 artigos para a produção desse estudo. Resultado e Discussão: O CA encontra-se como uma das majoritárias causas de morte globalmente, principalmente, carcinomas mamários e prostáticos. Ocorre que $61,2 \%$ dos diagnostico positivos de neoplasia mamaria, acontece quando a patologia encontrase em estado avançado. Não obstante o carcinoma prostático apesar de ser uma patologia comum, corrobora por uma elevada taxa de óbitos, consequente de diagnósticos tardios do mesmo devido a motivos de pré-conceito das ideologias dos homens. Conclusão: Por meio dessa pesquisa constatou-se que a demasiada importância do universo espiritual inerente de cada indivíduo nos processos de promoção de qualidade de vida.
\end{abstract}

Palavras-chave: Câncer; Cuidados paliativos; Espiritualidade; Terapia ocupacional; Oncologia terminal.

\begin{abstract}
Introduction: $\mathrm{CA}$ is a worldwide public health problem, which indiscriminately affects citizens without age, sex and race restrictions. The number of illnesses and deaths from this disease has multiplied over the years, and this fact was caused by multiple causes. When the therapeutic processes do not present proper results in the face of the evolution of the disease, so the situation becomes irreversible, in this context, the multidisciplinary health team should provide PC. Objective: This research was a literary review that exposed competent palliative care to Occupational Therapy in approaching people in a terminal ontological state and its application in line with the spirituality of the individual. Methodology: This is a literature review built from articles collected from online databases, specifically INCA, SCIELO, PUBMED and Google School. Articles from the years 2016 to 2022 were used, in total 24 articles were used for the production of this study. Results and Discussion: CA is one of the major causes of death globally, especially breast and prostate carcinomas. It happens that $61.2 \%$ of positive diagnoses of breast cancer occur when the pathology is in an advanced state. Despite prostatic carcinoma, despite being a common pathology, it corroborates a high rate of deaths, resulting from late diagnoses of the same due to reasons of preconception of men's ideologies. Conclusion: Through this research, it was found that the inherent spiritual universe of each individual is too important in the processes of promoting quality of life.
\end{abstract}

Keywords: Cancer; Palliative care; Spirituality; Occupational therapy; Terminal oncology.

\title{
Resumen
}

La CA es un problema de salud pública a nivel mundial, que afecta indiscriminadamente a ciudadanossinrestricciones de edad, sexo y raza. El número de enfermos y muertes por esta enfermedad se ha multiplicado a lo largo de losaños, y este hecho se produjo por múltiples causas. Cuandolosprocesosterapéuticos no presentan resultados adecuadosante laevolución de laenfermedad, por lo que lasituación se torna irreversible, enese contexto, el equipo multidisciplinario de saluddebe brindar CP. Objetivo: Esta investigaciónfue una revisiónliteraria que expusolos cuidados paliativos competentes a la Terapia Ocupacional enelabordaje de personas en estado ontológico terminal y suaplicaciónenconcordanciaconlaespiritualidaddel individuo. Metodología: Se trata de una revisión bibliográfica construida a partir de artículos recopilados de bases de datosen línea, específicamente INCA, SCIELO, PUBMED y Google School. Se utilizaron artículos de losaños 2016 a 2022, en total se utilizaron 24 artículos para laelaboración de este estudio. Resultados y Discusión: La CA es una de lasprincipales causas de muerte a nivel mundial, especialmente los carcinomas de mama y próstata. Sucede que el $61,2 \%$ de los diagnósticos positivos de cáncer de mama se dancuandolapatología se encuentraenun estado avanzado. A pesar de que el carcinoma de próstata, a pesar de ser una patologíacomún, corrobora una alta tasa de muertes, resultado de diagnósticos tardíos de lamisma por razones de preconcepción de lasideologías de loshombres. Conclusión: A través de esta investigación, se constató que el universo espiritual inherente a cada individuo es demasiado importante enlosprocesos de promoción de lacalidad de vida.

Palabras clave: Cáncer; Cuidados paliativos; Espiritualidade; Terapia ocupacional; Oncología terminal.

\section{Introdução}

O câncer (CA) é um problema de saúde pública mundial, que afeta indiscriminadamente os cidadãos sem restrição de idade, sexo e raça. O número de eivados e óbitos por essa doença multiplicou no decorrer dos anos, esse fato acarretou-se por múltiplas causas, como envelhecimento, crescimento populacional e crescimento dos fatores de risco (INCA, 2019). Além 
disso, as grandes potências mundiais trabalham com afinco, objetivando desenvolver novas vertentes de resguardar esse índice superior de indivíduos dessa enfermidade recorrente.

Essa enfermidade é uma das maiores causas de mortalidade mundialmente, consiste na multiplicação exacerbada de células tumorais, que afetam vários tecidos e órgãos no corpo humano (Siegel, Miller, Jemal, 2016). Essa comorbidade é ocasionada por copiosos fatores - internos e externos - pelo crescimento na prevalência de causas relacionadas a hábitos da urbanização, como sedentarismo, má alimentação e fatores ambientais. Além disto, a hereditariedade e erros na replicação de DNA, auxiliam no desenvolvimento dessas células (Tomasetti, Vogelstein, 2017; INCA, 2019).

Há diversos tipos de carcinomas, os de próstata e mama, são responsáveis pelas maiores taxas de óbitos entre senis (Santos et al., 2016). A incidência de CA de mama maligno ocorre, majoritariamente, em pessoas do sexo feminino na fase adulta, em seguida as mais afetadas são idosas. Posto que, o carcinoma na mama é uma patologia que inicialmente é assintomática, contribuindo para a diagnose tardia. Ademais, o reconhecimento precoce é o principal fator para reduzir a mortalidade devida essa anomalia (Filho, Miranda, 2020). No Nordeste, além do CA mamário, o prostático possui uma elevada incidência em idosos, acometendo a partir de 80 anos, englobando uma taxa de mortos 18 vezes, maior que, os de 60 a 64 anos (Carvalho, Paes, 2019).

Após diagnosticados, o indivíduo prosseguirá para o devido processo de tratamento, com radioterápico, iodoterapia, quimioterapia, terapia biológica e em alguns casos são correlacionados (Coropes et al., 2016). Com base nesses dados é possível prognosticar que o impacto do tumor na saúde funcional desses pacientes causara múltiplas desordens no cotidiano. Essas desordens funcionais provocam danos à capacidade de desempenhar as atividades de vida diárias (AVDs) nas suas relações sociais e situações financeiras (Freire et al., 2018).

A diagnose terminal se dar quando os processos terapêuticos não apresentam devidos resultados diante da evolução da doença, assim o quadro torna-se irreversível. Nesse contexto, a equipe multidisciplinar de saúde deverá provir cuidados paliativos (CP) (Coelho, Yankaskas, 2017; Marques, Pucci, 2021). Esses cuidados caracterizam-se como um intrincado sistema de cuidados multidisciplinares e interdisciplinares que compreendem as questões biomédicas, espirituais e psicossociais. Esses não estão centrados em postergar o óbito, porém fornecem cuidados necessários, com o intuito de garantir que as pessoas possam manter a independência e autonomia (Almeida et al., 2020; Marques, Pucci, 2021).

A espiritualidade apresenta correlação com a manutenção da vida e da saúde, influenciando fortemente no aspecto psicossocial. Logo, fundamental pondera em consideração, os cuidados com o enfermo, que se encontra em processo de reabilitação na fase crônica da doença. Por conseguinte, propicia o ser humano transpor o estado emocional e biológico, por meio de sua vivencia, exteriorizando o sentido profundo do seu existir e viver seu cotidiano (Benites, Neme, Santos, 2017). Essa pesquisa tem como finalidade, expor quais os CP competentes a Terapia Ocupacional (TO) na abordagem de pessoas em estado oncológico terminal, levando-se em respeito à espiritualidade no fator de demasiada importância para potencialização da qualidade e significado de vida.

\section{Metodologia}

Essa pesquisa tratou-se de uma revisão literária que expôs a correlação dos cuidados paliativos competentes a Terapia Ocupacional no trato aos pacientes oncológicos em estado crônico, com ênfase na influência da espiritualidade inerente ao paciente como objeto de promoção de qualidade de vida dos enfermos em seus momentos finais. Coletou-se artigos para construção desse estudo nos bancos de dados online, especificamente o Scientific Electronic Library Online (SCIELO), PUBMED, INCA e Google School. 
Retiraram-se como parâmetro de inclusão para o levantamento, os descritores em português: Câncer; Cuidados Paliativos; Espiritualidade; Terapia Ocupacional; Oncologia Terminal e em inglês: Cancer; PalliativeCare; Spirituality; OccupationalTherapy; Terminal Oncology. No propósito de tornar a busca mais específica, realizaram-se conexões entre os termos com a utilização dos operadores booleanos “AND”. Contendo como base publicações do período de 2016 a 2022 , que se referem a publicações com ênfase nos CP e mortalidade por diversos tipos de câncer. Levantou-se 271 artigos da SCIELO, 12 artigos da PUBMED e 1 do INCA, 2.030 artigos Google School, demonstrado no fluxograma a seguir (Tabela 1).

Utilizou-se desses, apenas 24 artigos, com limitação em português, inglês e espanhol, excluindo artigos com fuga de tema, desfoco do objetivo proposto nesse estudo, comentários e resumos de conferências. Toda pesquisa realizou-se de forma independente, os pesquisadores entrando em consenso do título a realização do estudo, analisando no programa Microsoft Excel versão Windows 10 (Tabela 2).

Tabela 1: Fluxograma com as etapas de análise realizadas para seleção dos artigos científicos.

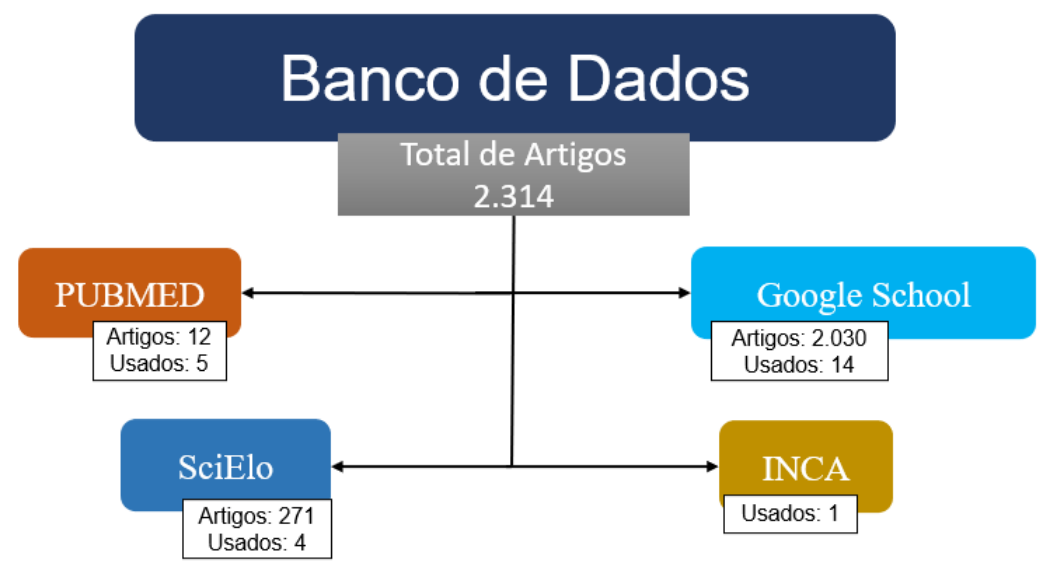

Total de Artigos

Usados

24

Fonte: Silva (2022).

A Tabela 2 apresenta uma listagem das principais publicações encontradas na busca ativa dos bancos de dados de periódicos na temática "A importância da espiritualidade como base para os cuidados paliativos a pacientes ontológicos terminais". 
Tabela 2: Fluxograma com as etapas de análise realizadas para seleção dos artigos científicos.

\begin{tabular}{|c|c|c|}
\hline Título do artigo & Autores e ano da pesquisa & Revista cientifica \\
\hline $\begin{array}{l}\text { Significados da espiritualidade para pacientes com câncer em cuidados } \\
\text { paliativos. }\end{array}$ & Benites, Neme, Santos, 2017. & SCIELO \\
\hline $\begin{array}{l}\text { Taxas de mortalidade por câncer corrigidas para os idosos dos estados do } \\
\text { Nordeste brasileiro. }\end{array}$ & Carvalho, Paes, 2019. & SCIELO \\
\hline Novos conceitos em cuidados paliativos na unidade de terapia intensiva. & Coelho, Yankaskas, 2017. & SCIELO \\
\hline Espiritualidade nos cuidados paliativos de pacientes oncológicos. & Marques, Pucci, 2021. & SCIELO \\
\hline Spirituality in palliative care: experiences of an interdisciplinary team. & Arrieiraet al, 2018. & PUBMED \\
\hline $\begin{array}{l}\text { What is spiritual care? Professional perspectives on the concept of spiritual } \\
\text { care identified through group concept mapping. }\end{array}$ & Hvidt et al, 2020 & PUBMED \\
\hline $\begin{array}{l}\text { Symptom clusters during palliative chemotherapy and their influence on } \\
\text { functioning and quality of life. }\end{array}$ & Rha, Lee, 2017. & PUBMED \\
\hline Cancerstatistics, 2016. CA: a cancerjournal for clinicians & Siegel, Miller, Jemal, 2016 & PUBMED \\
\hline $\begin{array}{l}\text { Stem cell divisions, somatic mutations, cancer etiology, and cancer } \\
\text { prevention. }\end{array}$ & Tomasetti et al., 2017 & PUBMED \\
\hline Mama e próstata. & INCA, 2019) & INCA \\
\hline $\begin{array}{l}\text { A Mamografia e seus Desafios: Fatores Socioeducacionais Associados ao } \\
\text { Diagnóstico Tardio do Câncer de Mama. }\end{array}$ & Assim, Mamede, 2016. & Google School \\
\hline $\begin{array}{l}\text { A Terapia Ocupacional nos Cuidados Paliativos Oncológicos-Revisão } \\
\text { Integrativa da Literatura }\end{array}$ & Botelho, 2019 & Google School \\
\hline $\begin{array}{l}\text { Ocupações e significados em cuidados paliativos oncológicos: o caso de" } \\
\text { Nobreza" em seu processo de finitude. }\end{array}$ & Calvalcante, Maués, 2018 & Google School \\
\hline $\begin{array}{l}\text { A assistência dos enfermeiros aos pacientes com câncer em fase terminal: } \\
\text { revisão integrativa }\end{array}$ & Coropes et al, 2016 & Google School \\
\hline Competências do Terapeuta Ocupacional em Cuidados Paliativos. & Costa et al., 2019 & Google School \\
\hline $\begin{array}{l}\text { Taxa de mortalidade em idosos acometidos por neoplasia maligna da mama e } \\
\text { próstata: nos anos de 2006-2016 na paraíba. }\end{array}$ & Dos Santos et al. 2017 & Google School \\
\hline $\begin{array}{l}\text { A relação entre o enfermeiro e o paciente nos cuidados paliativos } \\
\text { oncológicos. }\end{array}$ & De Almeida et al, 2020 & Google School \\
\hline $\begin{array}{l}\text { Descrição epidemiológica de pacientes diagnosticados com CA de mama no } \\
\text { Estado do Pará. }\end{array}$ & Vaz et al, 2020 & Google School \\
\hline $\begin{array}{l}\text { Avaliação da dor em paciente com câncer em cuidados paliativos à luz da } \\
\text { literatura. }\end{array}$ & Do Nascimento, 2017 & Google School \\
\hline $\begin{array}{l}\text { Promoção da espiritualidade através da musica como tratamento paliativo: um } \\
\text { relato de experiência }\end{array}$ & Dantas et al., 2017 & Google School \\
\hline $\begin{array}{l}\text { Qualidade de vida relacionada à saúde de pacientes com câncer em cuidados } \\
\text { paliativos. }\end{array}$ & Freire et al., 2018 & Google School \\
\hline $\begin{array}{l}\text { Perfil clínico-histológico das pacientes com carcinoma in situ de mama no } \\
\text { período de } 2003 \text { a } 2013 \text { em Manaus-AM. }\end{array}$ & Silva et al, 2020 & Google School \\
\hline Saúde preventiva com ênfase no câncer de próstata: uma revisão de literatura. & Veras et al, 2017 & Google School \\
\hline Espiritualidade e cuidados paliativos no tratamento de pacientes oncológicos. & Vidal et al, 2018 & Google School \\
\hline
\end{tabular}

Fonte: Silva (2022).

\section{Resultados e Discussões}

O câncer (CA) é uma patologia maliciosa e de avanço frenético, ao qual, desenvolve-se de maneira despercebida. No Brasil, anualmente, é verificado um crescimento do índice de mortalidade por essa enfermidade (Filho, Miranda, 2020). As pessoas que possuem um diagnóstico tardio dificultarão os seus recursos terapêuticos, logo a possibilidade do tratamento para atingir a cura, é quase nula. Ademais, com o impacto da diagnose, majoritariamente, afetam a sua saúde psicossocial. Nesses casos, é indispensável que o indivíduo seja assistido de forma holística pela equipe de saúde e receba os cuidados adequados.

Constatou-se que $61,2 \%$ dos resultados positivos de neoplasia mamaria, acontece quando encontra-se em estado avançado (Assis, Namede, 2016). No Brasil em 2017, sucedeu 16.724 óbitos devidos essa. Estima-se cerca de 66.280 novos casos de CA de mama por ano no período de 2020 a 2022. Representando uma estimativa de 61novos casos a cada 100 mil 
mulheres. Essa doença pode surgir por influência de diversos fatores, como genéticos, devido à mutação nos genes BRCA1 e BRCA2, hereditários, em razão de histórico familiar de pessoas com tumor no ovário, a menor pausa tardia em consequência de fatores da história reprodutiva hormonal, obesidade, sedentarismo e frequente exposição radiações ionizantes. Embora retenha condições de risco para essa enfermidade, a idade acima de 50 anos é considerada uma das mais relevantes (INCA, 2019).

O cancro na mama em mulheres é o carcinoma comum de células epiteliais, que pode classificar-se em lesões in situ ou invasoras. Esses tipos de tumores frequente são os ductais ou lobulares. O CA de próstata é o segundo com maior incidência em homens no mundo. No Brasil no ano de 2017, sucederam-se 15.391 mortes por essa (INCA, 2019). Em vários países menos desenvolvidos e em desenvolvimento, as estimativas mostram que o carcinoma prostático estará em primeiro lugar no número de óbito nas próximas décadas (Carvalho, Paes, 2019). Apesar de, caracterizar-se como rotineiro, ainda é pouco conhecido popularmente, mostrando-se como principal fator de risco para ocorrência a idade, após os 50 anos (INCA, 2019).

O câncer de próstata é usualmente chamado como o CA da terceira idade, devido corresponder a cerca de três quartos dos casos mundiais que ocorreram a partir dos 65 anos, é a sexta neoplasia que ocorre, comumente, no Brasil. Por múltiplos motivos, o homem evita os exames preventivos pelo constrangimento ao realiza-los ou pelo pré-conceito - consolidado em suas ideologias - pela necessidade de virilidade e intimidade física no exame, como toque retal. Contudo, as mulheres conseguem apresentar mais cuidados na sua saúde, com exames rotineiros e profiláticos anuais (Veras et al., 2017).

Contudo, os pacientes oncológicos em estágio terminal passam por cinco fases: negação, raiva, barganha, depressão e aceitação, necessariamente, não transitam por todas. A terminalidade é árdua, pois nela a certeza do óbito torna-se cada vez mais presente. Contudo a verdade, o diálogo e a decisões são direitos deles, tornando-se necessário que sejam garantidos (Coropes et al., 2016).

Diante desse quadro terminal, desenvolvem doenças como depressão, ansiedade, Transtorno do Déficit de Atenção e Hiperatividade (TDAH), entre outras. Dessa forma, o torna dificultoso o seu recurso terapêutico, impactando negativamente na sua recuperação ou qualidade de vida dos seus últimos dias. Contudo, é essencial que os profissionais que lidam com estagio terminais contenham o conhecimento sobre os CP, discutindo os objetivos com a família ou cuidadores. Assim, requerido que sejam respeitadas as vontades, as bases espirituais e sociais dos mesmos. Logo, a equipe multidisciplinar de saúde é essencial esclarecer a significância da terapia e dos cuidados, como melhor recurso terapêutico (Coelho, Yankaskas,2017; Rha, Lee, 2017).

Outrossim, o indivíduo oncológico terminal ver-se abalado psicossocialmente. Nessa situação, os CP são responsáveis por promover um ambiente confortável, além de uma conscientização a respeito da terminalidade. Entretanto, esses meios de tratamento não dispõem de impostos ao doente, sem a aprovação dos mesmos (Coelho,Yankaskas, 2017). Esses cuidados são de cunho humanístico, sem objetivo de cura, porém consistindo de fato no conjunto de ações, como higienização, apoio psicológico, ajuda acolhimento, alivio da dor e sofrimento, sintomas físicos, sociais, psicológicos e espirituais, com a finalidade de promover a manutenção de vida desses (Dantas et al.,2017; Nascimento, 2017).

A Terapia Ocupacional (TO) é uma profissão da área da saúde que geralmente é encarregado por atuar na reabilitação, com o uso da ocupação humana por meio de promoção de AVDs (Botelho, 2019). A mesma é de fundamental importância no tratamento paliativo oncológico, pois possibilita a manutenção da funcionalidade, a educação e orientação do acometido e de seu cuidador, possibilitando a elevada autonomia e identidade ocupacional, levando em conta a realidade causada pela enfermidade (Cavalcante, Maues, Castro, 2018).

As intervenções da TO em CP, a carcinoma ao grupo senil são de demasiada importância, pois se centralizam no monitoramento das condições físicas e emocionais, melhoria do desempenho de tarefas significativas, nas adaptações 
substanciais para a manutenção das funções físicas, cognitivas e sensoriais, compreendendo ao conforto físico, domínio da dor, fadiga e outras sintomatologias (Cavalcante, Maues, Castro, 2018).

O processo de TO é aplicado em cooperação com o paciente, familiares e cuidadores. Portanto, o profissional desenvolve com os mesmos uma relação de parceria, como base para o processo terapêutico. Refere-se como competência avaliar o cliente nos diversos contextos, objetivando projetar e construir produtos de apoio que possibilitam o máximo conforto, funcionalidade e autonomia. Essas intervenções consistem em aconselhar, ensinar e adaptar posicionamentos, desenvolver estratégias e utilização de produtos de apoio, realizar atividades de reabilitação, manutenção e estimulação das capacidades sensoriais, motoras e cognitivas, treino de técnicas de conservação de energia que garantem a continuidade ou recuperação do envolvimento ocupacional, com ênfase nos processos espirituais do indivíduo (Costa et al, 2019).

É imprescindível que o TO pondere o sentido da espiritualidade durante a prática dos $\mathrm{CP}$, logo que a espiritualidade abrange valores pessoais e íntimos, alicerçando-se no sentido da vida, promovendo o amadurecimento pessoal e da reflexão das experiências. Devido sua atuação com ocupação humana, mostra-se essencial a importância nesse contexto. Por conseguinte, a espiritualidade manifesta o sentido do indivíduo, assim como, a forma que vivencia seu cotidiano (Benites, Neme, Santos, 2017; Arrieira et al, 2018). Por isso, o terapeuta é incumbido de viabilizar os últimos desejos do enfermo, ou seja, as atividades significativas, de forma que estimule e desenvolva suas competências sociais, consideração as vontades, interessese a dignidade do doente (Costa et al, 2019; Arrieira et al, 2018; Hvidt et al, 2020).

Apesar de o enfermiço encontrar-se sem esperanças pela falta de expectativa de cura, a intervenção do TO oferta um bem-estar por meio da participação em atividades significativas (Cavalcante, Maues, Castro, 2018). De forma competente, esses profissionais contém as atribuições de preservar a identidade ocupacional, provir os treinos ao nível do descanso, sono, trabalho, lazer, educação, participação social, recorrer a técnicas para alivio de sintomas sem aplicação de fármacos, respeitarem as diferenças individuais, crenças, espiritualidade, hábitos, suas influencias na ocupação, treinar AVDs e instrumentais (Costa et al, 2019).

Nesta ótica é função do TO reconhecer as demandas espirituais dos pacientes e assim dar síntese a uma anamnese sobre o contexto espiritual do mesmo, ajudando o profissional a desenvolver novas formas de intervenção deste cunho, que se tornam capazes de viabilizar suporte as crenças do paciente, promovendo a possibilidade de rituais religiosos, assim, viabilizando uma postura acolhedora, fortalecendo as características humanísticas dos CP (Arrieira et al, 2018).

A espiritualidade em um termo geral abrange uma dimensão da personalidade, uma parte do indivíduo que visa atribuições de significados através das reações transcendentes vivenciadas por interconexões intrapessoais e interpessoais. Por sua vez, contêm influencia na saúde e qualidade de vida, ressaltando-se a sua importância na cura, reabilitação, ademais, facilitando o psicológico de pacientes crônicos (Vidal et al., 2018). As pessoas em processo terminal possuem dificuldades nas possibilidades de resolver questões diárias, por isso, os CP devem ajudá-los no desenvolvimento de oportunidades para o planejamento do óbito e como essa relação se dar junto à família, os preparando para o momento final (Cavalcante, Maues, Castro, 2018).

\section{Conclusão}

Destarte constata-se que no decorrer dos anos, a ocorrência de CA tornou-se comum, corroborando-se a problema de saúde pública mundialmente, devido caracterizar-se por uma doença silenciosa e de rápida multiplicação, facilitando, por conseguinte que ocorra um grande número de diagnósticos tardios, portando ressalta que a imprescindibilidade de investimento dos órgãos públicos de saúde, com a finalidade de promover uma melhor qualidade de tratamento a esses indivíduos que encontram em estado crônico. 
Outrossim, há uma necessidade emergente que haja um engajamento pelos profissionais da Terapia Ocupacional, afim de concluir pesquisas mais profundas no âmbito da administração de cuidados paliativos, com objetivo de prover manutenção de vida aos últimos dias dos pacientes. Por meio desse estudo constatou-se que, a demasiada importância do universo espiritual inerente de cada indivíduonos processos de promoção de qualidade de vida. Ressaltou-se a obrigação de considerar a espiritualidade e seus processos na administração desses cuidados.

\section{Referências}

Almeida, P. F. Barbosa, M. G. A. Santos, S. M. Silva, E. I. \& Oliveira Lins, S. R. (2020). A relação entre o enfermeiro e o paciente nos cuidados paliativos oncológicos. Brazilian Journal of Health Review, 3(2), 1465-1483. 10.34119/bjhrv3n2-011.

Arrieira, I. C. D. O., Thofehrn, M. B. Porto, A. R., Moura, P. M. M., Martins, C. L., \& Jacondino, M. B. (2018). Spirituality in palliativecare: experiencesofnainterdisciplinaryteam. Revista da Escola de Enfermagem da USP, 2. 10.1590/s1980-220x2017007403312

Assis, C. F. \& Mamede, M. (2016). A Mamografia e seus Desafios: Fatores Socioeducacionais Associados ao Diagnóstico Tardio do Câncer de Mama. Iniciação Científica Cesumar, 18(1), 63-72. 10.17765/1518-1243.2016v18n1p63-72.

Benites, A. C. Neme, C. M. B. \& Santos, M. A. (2017). Significados da espiritualidade para pacientes com câncer em cuidados paliativos. Estudos de Psicologia 34(4), 269-279. 10.1590/1982-02752017000200008)

Botelho, F. T. P. (2019). A Terapia Ocupacional nos Cuidados Paliativos Oncológicos-Revisão Integrativa da Literatura.U. Porto. https://hdl.handle.net/10216/126592.

Carvalho, J. B. \& Paes, N. A. (2019). Taxas de mortalidade por câncer corrigidas para os idosos dos estados do Nordeste brasileiro. Ciência Saúde Coletiva, 24, 3857-3866. 10.1590/1413-812320182410.03612018.

Cavalcante, A. C. Maués, N. C. S. \& Castro, G. G. A. (2018). Ocupações e significados em cuidados paliativos oncológicos: o caso de" Nobreza" em seu processo de finitude. Revista Família, Ciclos de Vida e Saúde no Contexto Social, 6(1), 140-151. 10.18554/REFACS.V6I1.1876.

Coelho, C. B. T.; \& Yankaskas, J. R. (2017). Novos conceitos em cuidados paliativos na unidade de terapia intensiva. Revista Brasileira de Terapia Intensiva, 29(2), 222-230. 10.5935/0103-507X.20170031.

Coropes, V. B. A. D. S. Valente, G. S. C. Oliveira, A. C. F. D. Paula, C. L. D. Souza, C. Q. S. D. \& Camacho, A. C. L. F. (2016). A assistência dos enfermeiros aos pacientes com câncer em fase terminal: revisão integrativa. Rev. enferm. UFPE on-line, 4920-4926. 10.5205/1981-8963-v10i6a11273p49204926-2016.

Costa, A. Monteiro, M. Salgado, R. \& Pedrosa, V. V. (2019). Competências do Terapeuta Ocupacional em Cuidados Paliativos. Associação Portuguesa de Terapeutas Ocupacionais; Associação Portuguesa de Cuidados Paliativos; Grupos de Interesses em Cuidados Paliativos e Terapia Ocupacional. http://hdl.handle.net/10400.8/4984.

De Lima Dantas, H. L. Silva, W. R. Paes, N. C. \& Comassetto. (2017). Promoção da espiritualidade através da musica como tratamento paliativo: um relato de experiência. Congresso Internacional de Envelhecimento Humano.

Dos Santos, M. M. S. de Holanda, G. S., Nogueira, G. S., Sarmento, W. M., \& Oliveira C. P. F. (2017). Taxa de mortalidade em idosos acometidos por neoplasia maligna da mama e próstata: nos anos de 2006-2016 na paraíba.CongressoInternacionalEnvelhecimento Humano. Anais $V$ CIEH... Campina Grande: Realize Editora.

Hvidt, N. C. Nielsen K. T. Kørup, A. K. Prinds, C. Hansen D. G. Viftrup D. T. AssingHvidt, E.Hammer E. R. Falkø E. Locher F. Boelsbjerg H. B. Wallin J. A. Thomsen K. F. Schrøder, K. Moestrup, L. Nissen, R. D. Stewart -Ferrer, S. Stripp T. K.Steenfeldt V. Ø. Søndergaard, J. \& Wæhrens E. E. (2020). What is spiritual care? Professional perspectives on the conceptof spiritual careidentifiedthroughgroupconceptmapping. BMJ aberto. 10(12), e042142. 10.1136/bmjopen-2020-042142.

Intituto Nacional Do Cancer (Brasil). (2021). Tema: Mama e próstata. https://www.inca.gov.br/sites/ufu.sti.inca.local/files/media/document/estimativa-2020incidencia-de-cancer-no-brasil.pdf.

Freire, M. E. M. Costa, S. F. G. D. Lima, R. A. G. D. \& Sawada, N. O. (2018). Qualidade de vida relacionada à saúde de pacientes com câncer em cuidados paliativos. Texto Contexto-Enfermagem, 27(2). 10.1590/0104-070720180005420016.

Marques, T. C. S. \& Pucci, S. H. M. (2021). Espiritualidade nos cuidados paliativos de pacientes oncológicos. Psicologia USP, 32. 10.1590/0103$6564 \mathrm{e} 200196$.

Nascimento, J. C. C. (2017). Avaliação da dor em paciente com câncer em cuidados paliativos à luz da literatura. Saúde Ciência Em Ação, 3(1), 11-26.

Rha, S. Y., \& Lee, J. (2016). Symptom clusters during palliative chemotherapy and their influence on functioning and quality of life. SupportiveCare in Cancer, 25(5), 1519-1527. 10.1007/s00520-016-3545-Z

Siegel, R. L. Miller, K. D. \& Jemal, A. (2016). Cancerstatistics. CA: a cancerjournal for clinicians, 66(1), 7-30.10.3322/caac.21332.

Silva, J. R. N. Silva, K. D. Nunes, G. P. S. Navarro, L. M. \& Pereira, H. F. B. E. S.A. (2020). Perfil clínico-histológico das pacientes com carcinoma in situ de mama no período de 2003 a 2013 em Manaus-AM. Sci. Amaz, 9(1), 26-30. 
Research, Society and Development, v. 11, n. 3, e2811326202, 2022

(CC BY 4.0) | ISSN 2525-3409 | DOI: http://dx.doi.org/10.33448/rsd-v11i3.26202

Tomasetti, C. Li, L. \& Vogelstein, B. (2017). Stem cell divisions, somatic mutations, cancer etiology, and cancer prevention. Science, 355(6331), 1330-1334. 10.1126/science.aaf9011.

Vaz, D. W. N.Oliveira, J. D. Filho, L. C. C. dos S. Miranda, T. L. K. S. Evangelista, H. I. \& Silva, J. B. (2020). Descrição epidemiológica de pacientes diagnosticados com CA de mama no Estado do Pará. Revista Brasileira De Educação E Saúde, 10(2), 86-90. 10.18378/rebes.v10i2.7788.

Veras, A. S. P. Aragão, F. B. A. Santos Pereira, J. F. Furtado, Q. R. Pereira, S. L. M. \& Silva Gomes, F. C. (2017). Saúde preventiva com ênfase no câncer de próstata: uma revisão de literatura. UningáJournal, 54(1), http://revista.uninga.br/index.php/uninga/article/view/7.

Vidal, A. L. P. Ferreira, A. C. Souza, L. G. P. Caetano, M. E. S. Chaves, T. A. \& Gonçalves, S. H. R. (2018). Espiritualidade e cuidados paliativos no tratamento de pacientes oncológicos. CIPEEX, 2, 1149-1160. 